\title{
SCHUR'S PARTITION THEOREM, COMPANIONS, REFINEMENTS AND GENERALIZATIONS
}

\author{
KRISHNASWAMI ALLADI AND BASIL GORDON
}

\begin{abstract}
Schur's partition theorem asserts the equality $S(n)=S_{1}(n)$, where $S(n)$ is the number of partitions of $n$ into distinct parts $\equiv 1,2(\bmod 3)$ and $S_{1}(n)$ is the number of partitions of $n$ into parts with minimal difference 3 and no consecutive multiples of 3 . Using a computer search Andrews found a companion result $S(n)=S_{2}(n)$, where $S_{2}(n)$ is the number of partitions of $n$ whose parts $e_{i}$ satisfy $e_{i}-e_{i+1} \geq 3,2$ or 5 according as $e_{i} \equiv 1,2$ or $3(\bmod 3)$. By means of a new technique called the method of weighted words, a combinatorial as well as a generating function proof of both these theorems are given simultaneously. It is shown that $S_{1}(n)$ and $S_{2}(n)$ are only two of six companion partition functions $S_{j}(n), j=1,2, \ldots, 6$, all equal to $S(n)$. A three parameter refinement and generalization of these results is obtained.
\end{abstract}

\section{INTRODUCTION}

The celebrated partition theorem which Schur [14] proved in 1926 is:

Theorem S. Let $S(n)$ denote the number of partitions of $n$ into distinct parts $\equiv 1,2(\bmod 3)$. Let $S_{1}(n)$ denote the number of partitions of $n$ with minimal difference 3 between parts and such that no two consecutive multiples of 3 occur as parts. Then

$$
S(n)=S_{1}(n) .
$$

Several proofs of Schur's theorem and extensions of it are known. Andrews [5] gave a proof using generating functions and subsequently obtained extensions [6], [7] with 3 replaced by a larger modulus $M$ and with gap conditions more complicated than those in Theorem S. In 1980, Bressoud [11] obtained a purely combinatorial proof and observed stronger correspondences between the partitions counted by $S(n)$ and $S_{1}(n)$.

In 1971 Andrews [8] found the following companion to Schur's theorem by a computer search:

Theorem A. Let $S_{2}(n)$ denote the number of partitions of $n$ in the form $n=$ $e_{1}+e_{2}+\cdots+e_{\nu}$ such that $e_{\ell}-e_{\ell+1} \geq 3,2$ or 5 if $e_{\ell} \equiv 1,2$ or $3(\bmod 3)$. Then

$$
S(n)=S_{1}(n)=S_{2}(n) .
$$

Received by the editors September 24, 1993. Presented to the Society on May 20,1993, at its 882nd meeting in DeKalb, Illinois.

1991 Mathematics Subject Classification. Primary 05A15, 05A17, 05A19, 11P81, 11 P83.

The first author's work was done at Pennsylvania State University while on sabbatical leave from the University of Florida during 1992-93. 
Andrews [8] gave a proof of Theorem A using generating functions in a manner similar to his previous proof of Theorem S. But the exact relationship between these two theorems remained unclear.

In a recent paper [1] we presented a new approach to Schur's theorem via what we called the method of weighted words. (See $\S 2$ for a description of this method and a summary of the results in [1].) This paper is a substantial extension of the ideas in [1] and has several important goals. Our first objective is to show that this method gives proofs of Theorems $\mathrm{S}$ and A simultaneously (see $\S 3$ ) and sets up a natural bijection between the partitions counted by $S_{1}(n)$ and $S_{2}(n)$. Our second, and more important, objective is to show that $S_{1}(n)$ and $S_{2}(n)$ are only two of six companion partition functions $S_{\mu}(n), \mu=$ $1,2, \ldots, 6$. (For the definitions of $S_{3}(n), S_{4}(n), S_{5}(n)$ and $S_{6}(n)$, see $\S 4$.) Our first main result is

Theorem 1. $S(n)=S_{\mu}(n), \mu=1,2, \ldots, 6$.

The gap conditions defining the partition functions $S_{\mu}(n), \mu=3,4,5,6$, are slightly more complicated and so did not show up in Andrews' computer search. For example, $S_{3}(n)$ is the number of partitions of $n$ in the form $n=e_{1}+e_{2}+\cdots+e_{\nu}$ such that

$$
e_{\ell}-e_{\ell+1} \begin{cases}=1 \text { or } \geq 3, & \text { if } e_{\ell} \equiv 1(\bmod 3), \\ \geq 2 \text { or } 6, & \text { if } e_{\ell} \equiv 2 \text { or } 3(\bmod 3) .\end{cases}
$$

The method of weighted words provides a suitable general setting in which the relationship between the six partition functions can be seen clearly. In $\S 5$ we explain the six equalities in Theorem 1 by means of a conservation law for the gaps. Our method shows that the six companion functions $S_{\mu}(n)$ actually correspond to the six ways in which the $q$-multinomial coefficient

$$
\left[\begin{array}{c}
i+j+k \\
i, j, k
\end{array}\right]=\frac{(q)_{i+j+k}}{(q)_{i}(q)_{j}(q)_{k}}
$$

can be expanded (see $\S 6$ ).

We set up natural bijections between the partitions counted by each of the functions $S_{\mu}(n)$. As a consequence of these bijections several refinements of Theorem 1 emerge, and we state one now.

Theorem 2. Let $S_{\mu}(n ; 3 m)$ denote the number of partitions counted by $S_{\mu}(n)$ with the added restriction that all parts are $\leq 3 m$. Then for all integers $n, m \geq$ 1 , we have

$$
S_{1}(n ; 3 m)=S_{2}(n ; 3 m)=\cdots=S_{6}(n ; 3 m) .
$$

For a proof of Theorem 2 see $\S 7$. What is more, we show that the $a-b-c$ generating function for these types of partitions counted by $S_{\mu}(n)$ are all equal, where the powers of the parameters $a, b$ and $c$ will represent the number of parts in residue classes 1,2 and $3(\bmod 3)$.

The third important objective in this paper is to show that the above theorems are special cases under the transformations

$$
\text { (dilation) } q \mapsto q^{3} \text { and (translations) } a \mapsto a q^{-2}, \quad b \mapsto b q^{-1} \text {. }
$$

From now on we will refer to the transformations in (1.3) as standard transformations. More generally, under the dilation $q \mapsto q^{M}$ and translations 
$a \mapsto a q^{\alpha-M}, b \mapsto b q^{\beta-M}$, one can get corresponding results with the modulus $M \geq 3$ replacing 3, where the powers of $a$ and $b$ keep track of parts in two arbitrary distinct residue classes $\alpha$ and $\beta(\bmod M)$. Thus the method of weighted words is acting at the base level without any dilations.

Recently, in collaboration with George Andrews, we have been able to extend the method of weighted words to obtain a new proof of deep partition theorems of Göllnitz ([13], Sätze 4.8 and 4.10) and to prove and refine a partition conjecture of Capparelli [12]. These will be discussed in forthcoming papers [2], [3]. Some remarks about these problems are made in $\S 8$.

We use the standard notation

$$
(a)_{n}=(a ; q)_{n}=(1-a)(1-a q) \cdots\left(1-a q^{n-1}\right)
$$

for an integer $n \geq 1$, and

$$
(a)_{\infty}=\lim _{n \rightarrow \infty}(a)_{n}, \quad \text { if }|q|<1 .
$$

As usual, null products have value 1 . The $q$-binomial coefficients are defined by

$$
\left[\begin{array}{c}
i+j \\
i, j
\end{array}\right]=\frac{(q)_{i+j}}{(q)_{i}(q)_{j}}
$$

and the multinomial coefficients are given in (1.2).

Given a partition $\pi$, we let $\sigma(\pi)$ denote the sum of its parts, $\lambda(\pi)$ its largest part and $\nu(\pi)$ the number of parts of $\pi$. Quite often we will be counting the number of parts of $\pi$ of a specific type, and this will be indicated by a subscript, eg: $\nu_{a}(\pi)$ will be the number of $a$-parts of $\pi$ (precise definitions will be given when necessary). Sometimes the parts will be arranged according to a specific lexicographic ordering (not necessarily the standard ordering of the positive integers). In such cases $\lambda(\pi)$ will denote the largest part of $\pi$ with respect to this ordering. We also adopt the convention that $j \equiv b(\bmod m)$ means that $j=b+\ell m$ with $j>0$ and $\ell \geq 0$.

Schur had originally stated the equality of three partition functions, $T(n)=$ $S(n)=S_{1}(n)$, where $T(n)$ is number of partitions of $n$ into parts $\equiv \pm 1$ (mod 6), repetitions allowed. However, in discussing $T(n)$, one does not get refinements; that is, it is not possible to keep track of the number of parts in specific residue classes as can be done with the partitions counted by $S(n)$ and $S_{1}(n)$. Hence we do not discuss the function $T(n)$ in this paper.

\section{THE METHOD OF WEIGHTED WORDS}

Let each integer $j \geq 2$ occur in three colors, red, blue and purple, and let the integer 1 occur only in two colors, red and blue. We introduce the symbols $a_{j}, b_{j}$ and $c_{j}$ to represent the integer (part) $j$ in colors red, blue and purple respectively, and sometimes we simply refer to $a_{j}$ as an $a$-part, $b_{j}$ as a $b$-part and $c_{j}$ as a $c$-part. We think of the letters $a, b$ and $c$ as representing free parameters. Under the standard transformations, the powers of the parameters $a, b$ and $c$ will represent the number of parts congruent to 1,2 and $3(\bmod 3)$ respectively in partitions counted by $S_{\mu}(n)$. However, in making the transition from $S_{\mu}(n)$ to $S(n)$, we will need to choose $c=a b$. That is why we think of $c$ having color purple $=$ red plus blue.

The gap between any two symbols representing colored integers is defined to be the absolute value of the difference between their subscripts. The gap 
therefore is a non-negative integer, without color. For example, the gap between $a_{5}$ and $c_{5}$ is 0 , and between $a_{5}$ and $b_{7}$ is 2 . The weight of a symbol is defined to be its subscript. For example, the weight of $b_{6}$ is 6 , and $b_{6}$ represents the integer 6 colored blue. Now in order to discuss partitions using these symbols, we need a lexicographic ordering of the symbols, and that is what we discuss next.

First we consider the following lexicographic ordering:

$$
a_{1} \prec b_{1} \prec c_{2} \prec a_{2} \prec b_{2} \prec c_{3} \prec a_{3} \prec b_{3} \prec \cdots \text {. }
$$

We shall refer to this as Scheme 1 . Now by a partition $\pi$, we mean a sum involving the symbols $a_{j}, b_{j}$ and $c_{j}$ where the symbols (parts) are arranged in decreasing order as given by Scheme 1. Instead of thinking of $\pi$ as a sum we may think of it as a word with symbols in decreasing order. By $\sigma(\pi)=n$ we mean that the sum of the weights of the symbols of $\pi$ is $n$. So $\pi$ is a partition of $n$ into colored integers. For example $\pi=a_{7}+c_{7}+b_{5}+b_{5}+a_{4}+$ $c_{4}+c_{2}+b_{1}+a_{1}+a_{1}$ is a partition of 37 because $\sigma(\pi)=37$. We let $\nu_{a}(\pi)$, $\nu_{b}(\pi)$ and $\nu_{c}(\pi)$ denote the number of $a$-parts, $b$-parts and $c$-parts of $\pi$. In this example $\nu_{a}(\pi)=4, \nu_{b}(\pi)=3$ and $\nu_{c}(\pi)=3$. In this example the largest part $\lambda(\pi)=a_{7}$.

Next we consider a partition $e_{1}+e_{2}+\cdots+e_{\nu}$ using symbols from Scheme 1 such that the gap between the symbols is $\geq 1$, with the added restriction that the gap between consecutive symbols $e_{\ell}, e_{\ell+1}$ is $>1$ if

$$
\left.\begin{array}{c}
e_{\ell} \text { is red, } e_{\ell+1} \text { is blue } \\
\text { or } e_{\ell} \text { is purple. }
\end{array}\right\}
$$

We will refer to this as a Type 1 partition. The following result was established in [1]:

Lemma 1. Let $G=G(i, j, k)$ be the generating function for all Type 1 partitions $\pi$ having $\nu_{a}(\pi)=i, \nu_{b}(\pi)=j$ and $\nu_{c}(\pi)=k$. That is,

$$
G=\sum_{\substack{\pi \text { of Type } 1 \\ \nu_{a}(\pi)=i, \nu_{b}(\pi)=j, \nu_{c}(\pi)=k}} q^{\sigma(\pi)} .
$$

Then

$$
G=\frac{q^{T_{i+j+k}+T_{k}}}{(q)_{i}(q)_{j}(q)_{k}},
$$

where $T_{m}=m(m+1) / 2$ is the mth triangular number.

We describe briefly the ideas behind the proof of Lemma 1, as this will be useful for more general results in the following sections.

Consider the generating function for all Type 1 partitions where the numbers $i, j, k$ of colored parts are specified and the order in which they occur is also given. Then the generating function can be computed as follows: First construct the minimal partition $\pi$ satisfying the restrictions. Then the required generating function is

$$
q^{\sigma(\pi)} /(q)_{\nu(\pi)}
$$

Since Type 1 partitions are given by lower bound gap conditions, the partitions in question are obtained by adding to $\pi$ all (ordinary) partitions $\rho$ with $\nu(\rho) \leq$ 
$\nu(\pi)$. Thus the expression in (2.3) is the required generating function. For example, if the colors occur in the order

$$
\begin{array}{lllll}
c & a & b & b & c
\end{array}
$$

then the minimal Type 1 partition is

$$
\pi=c_{8}+a_{6}+b_{4}+b_{3}+c_{2} .
$$

So $\sigma(\pi)=23, \nu(\pi)=5$, and the required generating function is

$$
q^{23} /(q)_{5} \text {. }
$$

In view of these observations, write the expression for $G$ in Lemma 1 as

$$
G=\frac{q^{T_{i+j+k}+T_{k}}}{(q)_{i+j+k}}\left[\begin{array}{c}
i+j+k \\
i, j, k
\end{array}\right]
$$

Since $\nu(\pi)=i+j+k$ for all partitions counted by $G$, we see from (2.3) and (2.4) that Lemma 1 is equivalent to the statement that the generating function $H=H(i, j, k)$ for all minimal partitions having $i$ red, $j$ blue and $k$ purple parts is

$$
H(i, j, k)=q^{T_{i+j+k}+T_{k}}\left[\begin{array}{c}
i+j+k \\
i, j, k
\end{array}\right] .
$$

To prove (2.5) consider the sets $A, B$ and $C$ of all partitions of Type 1 such that the smallest part is red, blue and purple respectively. Then consider the generating functions

$$
\begin{gathered}
H_{a}(i, j, k)=\sum_{\mu \in A} q^{\sigma(\mu)}, \quad H_{b}(i, j, k)=\sum_{\mu \in B} q^{\sigma(\mu)}, \\
H_{c}(i, j, k)=\sum_{\mu \in C} q^{\sigma(\mu)} .
\end{gathered}
$$

So

$$
H=H_{a}+H_{b}+H_{c} .
$$

It can be shown by induction on $i+j+k$ (see [1]) that

$$
\begin{aligned}
& H_{a}(i, j, k)=q^{T_{i+j+k}+T_{k}}\left[\begin{array}{c}
i+j+k-1 \\
i-1, j, k
\end{array}\right], \\
& H_{b}(i, j, k)=q^{T_{i+j+k}+T_{k}+i}\left[\begin{array}{c}
i+j+k-1 \\
i, j-1, k
\end{array}\right], \\
& H_{c}(i, j, k)=q^{T_{i+j+k}+T_{k}+i+j}\left[\begin{array}{c}
i+j+k-1 \\
i, j, k-1
\end{array}\right] .
\end{aligned}
$$

So (2.5) follows from (2.7) and (2.8) because of the recurrence (2.9)

$$
\left[\begin{array}{c}
i+j+k \\
i, j, k
\end{array}\right]=\left[\begin{array}{c}
i+j+k-1 \\
i-1, j, k
\end{array}\right]+q^{i}\left[\begin{array}{c}
i+j+k-1 \\
i, j-1, k
\end{array}\right]+q^{i+j}\left[\begin{array}{c}
i+j+k-1 \\
i, j, k-1
\end{array}\right]
$$

satisfied by the $q$-multinomial coefficients. 
Next, let $B_{1}(n ; i, j, k)$ denote the number of Type 1 partitions $\pi$ of $n$ having $\nu_{a}(\pi)=i, \nu_{b}(\pi)=j$, and $\nu_{c}(\pi)=k$. Then from Lemma 1 we see that

$$
\begin{aligned}
& \sum_{i, j, k, n} B(n ; i, j, k) a^{i} b^{j} c^{k} q^{n}=\sum_{i, j, k} a^{i} b^{j} c^{k} G(i, j, k) \\
& =\sum_{i, j, k} a^{i} b^{j} c^{k} \frac{q^{T_{i+j+k}+T_{k}}}{(q)_{i}(q)_{j}(q)_{k}} .
\end{aligned}
$$

Our next result is

Lemma 2. Let $r, s \geq 0$ be given integers. Then

$$
\sum_{0 \leq m \leq \min (r, s)} G(r-m, s-m, m)=\sum_{0 \leq m \leq \min (r, s)} \frac{q^{T_{r+s-m}+T_{m}}}{(q)_{r-m}(q)_{s-m}(q)_{m}}=\frac{q^{T_{r}+T_{s}}}{(q)_{r}(q)_{s}} .
$$

In [1] we give two proofs of Lemma 2, one of which is combinatorial. Lemma 2 has a nice combinatorial interpretation from which a refinement of Schur's theorem follows and we describe this next.

Note that

$$
\sum_{r, s} \frac{a^{r} b^{s} q^{T_{r}+T_{s}}}{(q)_{r}(q)_{s}}=(-a q)_{\infty}(-b q)_{\infty}=\sum_{n, r, s} V(n ; r, s) a^{r} b^{s} q^{n},
$$

where $V(n ; r, s)$ is the number of (vector) bi-partitions $\left(\pi_{1} ; \pi_{2}\right)$ of $n$ such that $\pi_{1}$ has $r$ distinct red parts and $\pi_{2}$ has $s$ distinct blue parts. So Lemma 2 is the assertion that the generating functions in (2.10) and (2.11) are equal when $c=a b$. In this case with

$$
i=r-m, \quad j=s-m, \quad k=m
$$

we have

$$
i+j+2 k=r+s .
$$

So we get the following combinatorial result:

Theorem 3. Let $t \geq 0$ be given and $V(n ; t)=\sum_{r+s=t} V(n ; r, s)$. Then

$$
V(n ; t)=\sum_{i+j+2 k=t} B(n ; i, j, k) .
$$

Theorem 3 gives a refinement of Theorem $\mathrm{S}$ under the standard transformations. More generally, under the dilation $q \mapsto q^{M}, a \mapsto a q^{\alpha-M}, b \mapsto b q^{\beta-M}$ applied to (2.10) and (2.11) we get the following refinement and generalization of Schur's theorem:

Theorem 4. Let $M \geq 3$ and $0<\alpha<\beta<M \leq \alpha+\beta$.

Let $A(n ; k)$ denote the number of partitions of $n$ into $k$ distinct parts congruent to $\alpha$ or $\beta(\bmod M)$.

Let $B(n ; k)$ denote the number of partitions of $n$ into $k$ parts $\equiv \alpha, \beta$ or $\alpha+\beta(\bmod M)$ such that

(i) the difference between any two parts is $\geq M$,

(ii) the difference between parts $\equiv(\alpha+\beta)(\bmod M)$ is $>M$,

(iii) the parts $\equiv \alpha+\beta(\bmod M)$ are counted twice. 
Then

$$
A(n ; k)=B(n ; k) \text {. }
$$

In [1] a more general form of Theorem 4 was stated by relaxing the condition $0<\alpha<\beta<M \leq \alpha+\beta$ to the simpler condition that $\alpha, \beta$ and $\alpha+\beta$ are incongruent $(\bmod M)$. The reason that condition $(2.12)$ enters into Theorem 3 is because, given a bi-partition of $n$ into $r$ distinct red parts and $s$ distinct blue parts, one takes $m$ of the red parts and $m$ of the blue parts to form $m$ purple parts, leaving behind $r-m$ red and $s-m$ blue parts. The combinatorial proof of Theorem $S$ given by Bressoud [11] gives a different bijection from the one we provide for Theorem 3 in [1]. In the next section we describe our combinatorial proof of Theorem 3, and by changing the ordering in the penultimate step of this proof, we can get Theorem A instead of Theorem S.

\section{Combinatorial proof of Theorems S AND A}

Under the standard transformation the symbols become

$$
a_{m}=3 m-2, \quad b_{m}=3 m-1, \quad c_{m+1}=(a b)_{m+1}=3 m,
$$

when $c=a b$. In this case the lexicographic ordering (2.1) of Scheme 1 yields the natural ordering

$$
1<2<3<4<\cdots
$$

of the positive integers.

Let $x_{m}=x_{m}^{(1)}$ denote the symbol occupying the $m$ th position in (2.1). That is, $x_{1}=a_{1}, x_{2}=b_{1}, x_{3}=c_{2}, \cdots$, and so on. Instead of defining Type 1 partitions by means of the gap conditions (2.2), we may define them equivalently as follows: Type 1 partitions are those of the form $x_{m_{1}}+x_{m_{2}}+\cdots+x_{m_{\nu}}$, where

$$
m_{\ell}-m_{\ell+1} \geq 3, \quad \text { with strict inequality if } x_{m_{\ell}} \text { is a } c \text {-part. }
$$

This is a direct translation of the difference conditions defining $S_{1}(n)$ to the more general situation involving weighted symbols. From now on we will refer to the inequalities in (3.2) as standard gap conditions.

In order to understand the difference conditions defining Andrews' function $S_{2}(n)$, consider another lexicographic ordering of the symbols, namely, Scheme 2 , given by

$$
a_{1} \prec c_{2} \prec b_{1} \prec a_{2} \prec c_{3} \prec b_{2} \prec a_{3} \prec c_{4} \prec b_{3} \prec \cdots .
$$

Under the standard transformations and with $c=a b$, Scheme 2 in (3.3) yields the following different ordering of the positive integers:

$$
1 \prec 3 \prec 2 \prec 4 \prec 6 \prec 5 \prec 7 \prec 9 \prec 8 \prec \cdots
$$

Now let $x_{m}^{(2)}$ denote the symbol occupying the $m$ th position in (3.4). Then the difference conditions defining Andrews' function $S_{2}(n)$ are equivalent to the statement that we consider partitions of the form $x_{m_{1}}^{(2)}+x_{m_{2}}^{(2)}+\cdots+x_{m_{\nu}}^{(2)}$, where the $x_{m_{\ell}}=x_{m_{\ell}}^{(2)}$ satisfy the standard gap conditions (3.2). More generally, if $x_{m}^{(2)}$ denotes the symbol occupying the $m$ th position in (3.3), let a Type 2 partition be one of the form $x_{m_{1}}^{(2)}+\cdots+x_{m_{\nu}}^{(2)}$, with $x_{m_{\ell}}=x_{m_{\ell}}^{(2)}$ satisfying (3.2). Also let $B_{2}(n ; i, j, k)$ denote the number of Type 2 partitions $\pi$ of $n$ having $\nu_{a}(\pi)=i, \nu_{b}(\pi)=j$ and $\nu_{c}(\pi)=k$. We then have 
Theorem 5. Let $n>0$ and $i, j, k \geq 0$ be integers. Then

$$
B_{1}(n ; i, j, k)=B_{2}(n ; i, j, k) .
$$

Theorem $\mathrm{A}$ is a consequence of Theorem 5 under the standard transformations.

We now discuss a combinatorial method which gives a bijective proof of Theorem A and more. The proof given here is an extension of the method in [1], which itself was based on earlier ideas of Bressoud [10].

Let $r, s$ be given. We start with a bipartition $\left(\pi_{1} ; \pi_{2}\right)$ counted by $V(n ; r, s)$. So $\nu\left(\pi_{1}\right)=r, \nu\left(\pi_{2}\right)=s$. In what follows, several steps will be given and illustrated with

$$
\pi_{1}=a_{7}+a_{6}+a_{3}+a_{2}+a_{1} \text { and } \pi_{2}=b_{13}+b_{12}+b_{8}+b_{7}+b_{5}+b_{4}+b_{2} \text {. }
$$

Step 1 . Decompose $\pi_{2}$ into $\pi_{4}+\pi_{5}$, where $\pi_{4}$ has the parts of $\pi_{2}$ which are $\leq r$ and $\pi_{5}$ has the remaining parts. Say, $\nu\left(\pi_{4}\right)=m$. So $\nu\left(\pi_{5}\right)=s-m$, and

$$
\pi_{4}=b_{5}+b_{4}+b_{2}, \quad \pi_{5}=b_{13}+b_{12}+b_{8}+b_{7} .
$$

Step 2. Consider the conjugate of the Ferrer's graph of $\pi_{4}$ and circle the bottom node in each column. Denote this graph by $\pi_{4}^{*}$. Construct the Ferrer's graph $\pi_{6}$, where the number of nodes in each row of $\pi_{6}$ is the sum of the number of nodes in the corresponding rows of $\pi_{1}$ and $\pi_{4}^{*}$. The parts of $\pi_{6}$ ending in the circled nodes are colored purple, and the remaining parts of $\pi_{6}$ are red. Thus $\pi_{6}$ has $r-m$ red parts and $m$ purple parts, and

$$
\pi_{6}=\pi_{1}+\pi_{4}^{*}=a_{10}+c_{9}+a_{5}+c_{4}+c_{2} .
$$

Step 3. Write the parts of $\pi_{5}$ in a column in descending order, and below them write the parts of $\pi_{6}$ in descending order. Draw a line to separate the parts of $\pi_{5}$ and $\pi_{6}$.

Step 4. Subtract 0 from the bottom element, 1 from the next element above that, 2 from the one above that, $\cdots$, and display the new values and the subtracted values in two adjacent columns $C_{1} \mid C_{2}$. The elements of $C_{2}$ have no color, while those of $C_{1}$ retain the color of the parts from which they were derived.

\begin{tabular}{ccc|c} 
Step 3 & & \multicolumn{2}{c}{ Step 4 } \\
$\begin{array}{ccc}\pi_{5} / \pi_{6} \\
b_{13}\end{array}$ & & $\frac{C_{1}}{b_{5}}$ & $\frac{C_{2}}{8}$ \\
$b_{12}$ & & $b_{5}$ & 7 \\
$b_{8}$ & & $b_{2}$ & 6 \\
$b_{7}$ & & $\underline{b_{2}}$ & 5 \\
$a_{10}$ & & $a_{6}$ & 4 \\
$c_{9}$ & & $c_{6}$ & 3 \\
$a_{5}$ & & $a_{3}$ & 2 \\
$c_{4}$ & & $c_{3}$ & 1 \\
$c_{2}$ & & $c_{2}$ & 0
\end{tabular}


Step 5 (Penultimate Step). Rearrange the entries of $C_{1}$ in descending order according to Scheme 1 or Scheme 2, to form a column $C_{1}^{R}$.

Step 6 (Final Step). Add the corresponding elements of $C_{1}^{R}$ and $C_{2}$ to get a partition counted by $B_{1}(n ; r-m, s-m, m)$ or $B_{2}(n ; r-m, s-m, m)$ depending upon which ordering was chosen at the penultimate step.

\section{Scheme 1}

\begin{tabular}{c|ccc|cc}
\multicolumn{2}{c}{ Step 5 } & $\underline{\text { Step 6 }}$ & \multicolumn{2}{c}{$\underline{\text { Step } 5}$} & $\underline{\text { Step 6 }}$ \\
$\frac{C_{1}^{R}}{a_{6}}$ & $\frac{C_{2}}{8}$ & $\frac{\pi_{3}}{a_{14}}$ & $\frac{C_{1}^{R}}{a_{6}}$ & $\frac{C_{2}}{8}$ & $\frac{\pi_{3}}{a_{14}}$ \\
$c_{6}$ & 7 & $c_{13}$ & $b_{5}$ & 7 & $b_{12}$ \\
$b_{5}$ & 6 & $b_{11}$ & $b_{5}$ & 6 & $b_{11}$ \\
$b_{5}$ & 5 & $b_{10}$ & $c_{6}$ & 5 & $c_{11}$ \\
$a_{3}$ & 4 & $a_{7}$ & $a_{3}$ & 4 & $a_{7}$ \\
$c_{3}$ & 3 & $c_{6}$ & $b_{2}$ & 3 & $b_{5}$ \\
$b_{2}$ & 2 & $b_{4}$ & $b_{2}$ & 2 & $b_{4}$ \\
$b_{2}$ & 1 & $b_{3}$ & $c_{3}$ & 1 & $c_{4}$ \\
$c_{2}$ & 0 & $c_{2}$ & $c_{2}$ & 0 & $c_{2}$
\end{tabular}

Every one of these steps is a one-to-one correspondence. So this procedure shows two things:

(i) Let $r, s$ be given. Then

$$
V(n ; r, s)=\sum_{m} B_{1}(n ; r-m, s-m, m)=\sum_{m} B_{2}(n ; r-m, s-m, m)
$$

$$
\begin{aligned}
& B_{1}(n ; i, j, k)=B_{2}(n ; i, j, k), \\
& \quad \text { taking } r-m=i, s-m=j, m=k .
\end{aligned}
$$

Theorem 5 is a consequence of (3.5). Theorems $\mathrm{S}$ and $\mathrm{A}$ are consequences of (3.4) under the standard transformations.

To simply get a bijection between the partitions counted by $B_{1}(n ; i, j, k)$ and $B_{2}(n ; i, j, k)$, proceed from Step 6 to Step 5, replace the Scheme 1 ordering in $C_{1}^{R}$ by the Scheme 2 ordering, and return to Step 6.

\section{SIX SCHEMES AND SIX COMPANIONS TO SCHUR}

Scheme 1 was generated by the standard ordering $a_{1} \prec b_{1} \prec c_{2}$ while Scheme 2 was generated by a different ordering $a_{1} \prec c_{2} \prec b_{1}$. More generally, we see that there are six schemes given by the six permutations of the symbols $a_{1}, b_{1}$ 
and $c_{2}$. They are

Scheme 1: $\quad a_{1} \prec b_{1} \prec c_{2} \prec a_{2} \prec b_{2} \prec c_{3} \prec \cdots$

Scheme 2: $\quad a_{1} \prec c_{2} \prec b_{1} \prec a_{2} \prec c_{3} \prec b_{2} \prec \cdots$

Scheme 3: $\quad c_{2} \prec a_{1} \prec b_{1} \prec c_{3} \prec a_{2} \prec b_{2} \prec \cdots$

Scheme 4: $\quad b_{1} \prec a_{1} \prec c_{2} \prec b_{2} \prec a_{2} \prec c_{3} \prec \cdots$

Scheme 5: $\quad b_{1} \prec c_{2} \prec a_{1} \prec b_{2} \prec c_{3} \prec a_{2} \prec \cdots$

Scheme 6: $\quad c_{2} \prec b_{1} \prec a_{1} \prec c_{3} \prec b_{2} \prec a_{2} \prec \cdots$

Actually, only three of the six schemes are essentially different, because Schemes 4, 5 and 6, are obtained from Schemes 1, 2 and 3 by interchanging the roles of $a$ and $b$. But there are certain advantages in discussing all six schemes, as will be seen in the sequel.

Next, let $x_{m}^{(\mu)}$ denote the symbol occupying position $m$ in Scheme $\mu, \mu=$ $1,2, \cdots, 6$. By a partition $\pi$ of Type $\mu$ we mean an expression $x_{m_{1}}^{(\mu)}+x_{m_{2}}^{(\mu)}+$ $\cdots+x_{m_{\nu}}^{(\mu)}$, where the $x_{m_{\ell}}=x_{m_{\ell}}^{(\mu)}$ satisfy the standard gap conditions (3.2). Let $B_{\mu}(n ; i, j, k)$ denote the number of partitions $\pi$ of $n$ of Type $\mu$ such that $\nu_{a}(\pi)=i, \nu_{b}(\pi)=j$ and $\nu_{c}(\pi)=k$. Then the combinatorial method of $\S 3$ shows that

Theorem 6. Let $n \geq 1$ and $i, j, k \geq 0$ be given integers. Then

$$
B_{1}(n ; i, j, k)=B_{2}(n ; i, j, k)=\cdots=B_{6}(n ; i, j, k) \text {. }
$$

Theorem 7. Let $r, s \geq 0$ be given integers. Then for $\mu=1,2, \cdots, 6$, we have

$$
V(n ; r, s)=\sum_{0 \leq m \leq \min (r, s)} B_{\mu}(n ; r-m, s-m, m) .
$$

Consequently, if $r+s=t$, then

$$
V(n ; t)=\sum_{i+j+2 k=t} B_{\mu}(n ; i, j, k) .
$$

Theorem 7 is a consequence of Theorem 6 and (3.4). To see the equality of the partition functions $B_{\mu}(n ; i, j, k)$ and $B_{\omega}(n ; i, j, k)$ one may use the ideas of $\S 3$ to construct a bijection $\mathscr{F}_{\mu, \omega}$ between the partitions $\pi_{\mu}$ counted by $B_{\mu}$ and the partitions $\pi_{\omega}$ counted by $B_{\omega}$. Indeed, if $\pi_{\mu}$ is given, then write the parts of $\pi_{\mu}$ in a column in descending order as in Step 6. Now subtract $0,1,2, \cdots$, in succession from the bottom part upwards to get the columns $C_{1}^{R} \mid C_{2}$ of Step 5. The entries of $C_{1}^{R}$ will be in descending order according to Scheme $\mu$. Rearrange the entries of $C_{1}^{R}$ according to the order given by Scheme $\omega$ and add back on the corresponding elements of $C_{2}$. Now we are back in Step 6 with a partition $\pi_{\omega}$ counted by $B_{\omega}$. Thus

$$
\mathscr{F}_{\mu, \omega}\left(\pi_{\mu}\right)=\pi_{\omega}
$$

and the construction is reversible. That is,

$$
\mathscr{F}_{\mu, \omega}^{-1}=\mathscr{F}_{\omega, \mu},
$$

establishing the equality claimed in Theorem 6. 
Under the standard transformations, the gap conditions (3.2) defining the Type $\mu$ partitions, $\mu=1,2, \cdots, 6$, become the following: We are now counting partitions of the form $e_{1}+e_{2}+\cdots+e_{\nu}$, where the $e_{\ell}$ are (ordinary) positive integers satisfying the difference conditions given by

(4.7) Type 1: $\quad e_{\ell}-e_{\ell+1} \geq 3,3$, or $4, \quad$ if $e_{\ell} \equiv 1,2$, or $3(\bmod 3)$.

(4.8) Type 2: $\quad e_{\ell}-e_{\ell+1} \geq 3,2$, or 5, if $e_{\ell} \equiv 1,2$, or $3 \quad(\bmod 3)$.

$$
\text { Type 3: } \left.\begin{array}{rl}
\quad e_{\ell}-e_{\ell+1} & =1 \text { or } \geq 3, \quad \text { if } e_{\ell} \equiv 1(\bmod 3) \\
\geq 2 \text { or } 6, \quad \text { if } e_{\ell} \equiv 2 \text { or } 3(\bmod 3)
\end{array}\right\} .
$$$$
\text { Type 4: } \left.\begin{array}{rl}
e_{\ell}-e_{\ell+1} & \geq 2 \text { or } 4, \quad \text { if } e_{\ell} \equiv 1 \text { or } 3(\bmod 3) \\
& =3 \text { or } \geq 5, \quad \text { if } e_{\ell} \equiv 2(\bmod 3)
\end{array}\right\} .
$$

Type 5: $\quad e_{\ell}-e_{\ell+1} \geq 1, \quad$ if $e_{\ell} \equiv 1(\bmod 3)$

$$
\left.\begin{array}{l}
=3 \text { or } \geq 5, \quad \text { if } e_{\ell} \equiv 2(\bmod 3) \\
=4 \text { or } \geq 6, \quad \text { if } e_{\ell} \equiv 3(\bmod 3)
\end{array}\right\} \text {. }
$$

$$
\text { Type 6: } \left.\begin{array}{rl}
e_{\ell}-e_{\ell+1} & \geq 1 \text { or } 6, \quad \text { if } e_{\ell} \equiv 1 \text { or } 3(\bmod 3) \\
& =2,3 \text { or } \geq 5, \quad \text { if } e_{\ell} \equiv 2 \text { or } 3(\bmod 3)
\end{array}\right\} .
$$

Note that the difference conditions in (4.7) are precisely those defining $S_{1}(n)$ in Theorem $S$ and the conditions in (4.8) are the same as those given by Andrews for $S_{2}(n)$ in Theorem A.

Next let $S_{\mu}(n ; i, j, k)$ denote the number of Type $\mu$ partitions of $n$ having $i$ parts $\equiv 1(\bmod 3), j$ parts $\equiv 2(\bmod 3)$ and $k$ parts $\equiv 3(\bmod 3)$. Then using Theorems 6 and 7 and the standard transformations, Theorems S, A and 1 in $\S 1$ can be improved to:

Theorem 8. Given integers $n \geq 1$ and $i, j, k \geq 0$,

$$
S_{1}(n ; i, j, k)=S_{2}(n ; i, j, k)=\cdots=S_{6}(n ; i, j, k) \text {. }
$$

Theorem 9. Given integers $n \geq 1$ and $r, s \geq 0$, let $S(n ; r, s)$ denote the number of partitions of $n$ into $r$ distinct parts $\equiv 1(\bmod 3)$ and $s$ distinct parts $\equiv 2(\bmod 3)$. Then

$$
S(n ; r, s)=\sum_{0 \leq m \leq \min (r, s)} S_{\mu}(n ; r-m, s-m, m), \quad \mu=1,2, \cdots, 6 .
$$

\section{A CONSERVATION LAW FOR GAPS}

The gap conditions in (2.2) which define the Type 1 partitions can be put in the following matrix form:

Type 1:

\begin{tabular}{c|ccc} 
& $a$ & $b$ & $c$ \\
\hline$a$ & 1 & 1 & 2 \\
$b$ & 2 & 1 & 2 \\
$c$ & 1 & 1 & 2
\end{tabular}


The entries in this matrix are the minimum gaps allowed in Type 1 partitions. To be more precise, the entries $2,1,2$ in the second row have the following interpretation. If the weight of a certain $b$ part is $w$ and if the next bigger part is an $a$-part, then that $a$-part has weight $\geq w+2$. If the next bigger part is a $b$-part, its weight is $\geq w+1$, and if it is a $c$-part, its weight is $\geq w+2$.

For Type 2 partitions, the gap conditions defining them can be embodied in the matrix

\begin{tabular}{cc|ccc} 
& & $a$ & $b$ & $c$ \\
\hline Type 2: & $a$ & 1 & 1 & 2 \\
& $b$ & 2 & 1 & 3 \\
& $c$ & 1 & 0 & 2
\end{tabular}

There are two similarities between these two matrices. To describe this let $x$ and $y$ take values $a, b$ and $c$ and let $\gamma_{1}(x, y)$ (resp. $\left.\gamma_{2}(x, y)\right)$ denote the entry corresponding to row $x$ and column $y$ in the matrix of Type 1 (resp. Type 2). (For example $\gamma_{1}(b, a)=2$ and $\gamma_{2}(b, c)=3$.) Then the diagonal entries of the two matrices are the same, namely

$$
\gamma_{1}(a, a)=\gamma_{2}(a, a)=1, \quad \gamma_{1}(b, b)=\gamma_{2}(b, b)=1, \quad \gamma_{3}(c, c)=\gamma_{2}(c, c)=1 .
$$

The second similarity is that

$$
\gamma_{\mu}(x, y)+\gamma_{\mu}(y, x)=3, \quad \mu=1,2, \quad x \neq y .
$$

In other words, the conservation law is that the sum of the gaps between $x, y$ and $y, x$ is $\geq 3$.

The matrices defining the other four types of partitions are given by

Type 3:

\begin{tabular}{c|ccc} 
& $a$ & $b$ & $c$ \\
\hline$a$ & 1 & 1 & 3 \\
$b$ & 2 & 1 & 3 \\
$c$ & 0 & 0 & 2
\end{tabular}

Type 4:

\begin{tabular}{l|lll} 
& $a$ & $b$ & $c$ \\
\hline$a$ & 1 & 2 & 2 \\
$b$ & 1 & 1 & 2 \\
$c$ & 1 & 1 & 2
\end{tabular}

Type 5:

Type 6:

\begin{tabular}{c|ccc} 
& $a$ & $b$ & $c$ \\
\hline$a$ & 1 & 2 & 3 \\
$b$ & 1 & 1 & 2 \\
$c$ & 0 & 1 & 2 \\
& $a$ & $b$ & $c$ \\
\hline$a$ & 1 & 2 & 3 \\
$b$ & 1 & 1 & 3 \\
$c$ & 0 & 0 & 2
\end{tabular}


So if $\gamma_{\mu}(x, y)$ denotes the entry corresponding to row $x$ and column $y$ in the matrix for Type $\mu, \mu=1,2, \cdots, 6$, then we have:

$$
\gamma_{\mu}(a, a)=\gamma_{\mu}(b, b)=1, \quad \gamma_{\mu}(c, c)=2, \quad \mu=1,2, \cdots, 6,
$$

extending (5.1). In addition we have the conservation law

$$
\gamma_{\mu}(x, y)+\gamma_{\mu}(y, x)=3, \quad \mu=1,2, \cdots, 6, x \neq y .
$$

Under the standard transformations, the conservation laws (5.3) and (5.4) may be recast in the form given below:

\section{The average values of the lower bounds for the differences}

depending on the residue class of $e_{i}(\bmod 3)$ add up to ten.

More precisely, the lower bounds 3, 3, 4 in (4.7) defining $S_{1}(n)$ add up to 10 . Similarly the lower bounds $3,2,5$ of Andrews in (4.8) also add up to 10. For Type 3 partitions counted by $S_{3}(n)$, the average of 1 and 3 is 2 , and so the sum of the averages of the lower bounds in (4.9) is $2+2+6=10$. Similarly in (4.10), with 4 as the average of 3 and 5 we have $2+4+4=10$. Note that in (4.11) with 3 and 5 having average 4 , and 4 and 6 having average 5 , we have $1+4+5=10$. Finally in (4.11), interpreting the average between 2,3 and 5 as 3 , we have $1+6+3=10$. Thus $(5.5)$ has been verified.

\section{MUltinOMial COEFFICIENT EXPANSIONS}

The proof of Lemma 1 we presented in $\S 2$ made use of the recurrence (2.9) for the $q$-multinomial coefficients. The function $G$ in Lemma 1 is the generating function of all Type 1 partitions using $i$ red, $j$ blue and $k$ purple parts. In view of Theorem 6 , the generating function will be identical for all Type $\mu$ partitions, $\mu=1,2, \cdots, 6$, using the same number of parts in the three colors. In this section we give a direct proof of this fact (i.e. without the use of Theorem 6 ) by means of all six recurrences satisfied by the $q$-multinomial coefficients. Thus Theorem 6 is a consequence of the results of this section.

The $q$-multinomial coefficient $\left[\begin{array}{c}i+j+k \\ i, j, k\end{array}\right]$ satisfies a total of six basic recurrences depending upon the order in which we reduce each of the letters $i, j$ and $k$ by one. One recurrence is already given in (2.9). Two other recurrences are (6.1)

$$
\left[\begin{array}{c}
i+j+k \\
i, j, k
\end{array}\right]=\left[\begin{array}{c}
i+j+k-1 \\
i-1, j, k
\end{array}\right]+q^{i}\left[\begin{array}{c}
i+j+k-1 \\
i, j, k-1
\end{array}\right]+q^{i+k}\left[\begin{array}{c}
i+j+k-1 \\
i, j-1, k
\end{array}\right]
$$

and

$$
\left[\begin{array}{c}
i+j+k \\
i, j, k
\end{array}\right]=\left[\begin{array}{c}
i+j+k-1 \\
i, j, k-1
\end{array}\right]+q^{k}\left[\begin{array}{c}
i+j+k-1 \\
i-1, j, k
\end{array}\right]+q^{k+i}\left[\begin{array}{c}
i+j+k-1 \\
i, j-1, k
\end{array}\right] .
$$

There are three more recurrences which we have not written down here.

Next, let $H_{a}^{(\mu)}(i, j, k), H_{b}^{(\mu)}(i, j, k)$, and $H_{c}^{(\mu)}(i, j, k)$ denote the generating function of all minimal Type $\mu$ partitions using $i$ red, $j$ blue and $k$ purple parts, such that the smallest part is $a_{1}, b_{1}$ and $c_{2}$ respectively. Then these generating functions can be computed by induction on $i+j+k$ in a manner similar to (2.8), as will be described presently.

For Type 1 partitions the starting triple for Scheme 1 ordering is

$$
\text { Scheme 1: } \quad a_{1} \prec b_{1} \prec c_{2} \text {. }
$$


Hence the generating functions $H_{a}^{(1)}, H_{b}^{(1)}$ and $H_{c}^{(1)}$ in (2.8) are given in terms of $q$-multinomial coefficients obtained by replacing $i$ by $i-1, j$ by $j-1$ and $k$ by $k-1$, in that order.

For Type 2 partitions the starting triple for Scheme 2 ordering is

$$
\text { Scheme 2: } \quad a_{1} \prec c_{2} \prec b_{1} \text {. }
$$

So, in this case we have

$$
\begin{aligned}
& H_{a}^{(2)}(i, j, k)=q^{T_{i+j+k}+T_{k}}\left[\begin{array}{c}
i+j+k-1 \\
i-1, j, k
\end{array}\right], \\
& H_{c}^{(2)}(i, j, k)=q^{T_{i+j+k}+T_{k}+i}\left[\begin{array}{c}
i+j+k-1 \\
i, j, k-1
\end{array}\right], \\
& H_{b}^{(2)}(i, j, k)=q^{T_{i+j+k}+T_{k}+i+k}\left[\begin{array}{c}
i+j+k-1 \\
i, j-1, k
\end{array}\right] .
\end{aligned}
$$

Equation (6.3) can be established by induction on $i+j+k$. In Scheme 2, since $a_{1}$ occurs first followed by $c_{2}$ and then by $b_{1}$, we reduced the letters $i, j, k$ by 1 in that order in (6.3). From (6.1) and (6.3) it follows that

$$
H_{a}^{(2)}+H_{b}^{(2)}+H_{c}^{(2)}=H=\frac{G}{(q)_{i+j+k}}
$$

with $H$ as in (2.5). Thus Scheme 2 corresponds to the recurrence (6.1) just as Scheme 1 corresponds to (2.9).

Similarly, since the starting triple for Scheme 3 ordering is

$$
\text { Scheme 3: } \quad c_{2} \prec a_{1} \prec b_{1},
$$

we have

$$
\begin{aligned}
& H_{c}^{(3)}(i, j, k)=q^{T_{i+j+k}+T_{k}}\left[\begin{array}{c}
i+j+k-1 \\
i, j, k-1
\end{array}\right], \\
& H_{a}^{(3)}(i, j, k)=q^{T_{i+j+k}+T_{k}+k}\left[\begin{array}{c}
i+j+k-1 \\
i-1, j, k
\end{array}\right], \\
& H_{b}^{(3)}(i, j, k)=q^{T_{i+j+k}+T_{k}+k+i}\left[\begin{array}{c}
i+j+k-1 \\
i, j-1, k
\end{array}\right] .
\end{aligned}
$$

This is easily established by induction on $i+j+k$. From (6.5) and (6.2) it follows that

$$
H_{a}^{(3)}+H_{b}^{(3)}+H_{c}^{(3)}=H,
$$

and so Scheme 3 corresponds to the third recurrence (6.2) for $q$-multinomial coefficients.

The treatment of the generating functions $H_{a}^{(\mu)}, H_{b}^{(\mu)}$ and $H_{c}^{(\mu)}$, for $\mu=$ 4, 5, 6 is similar. We record the ideas of this section in the form of

Theorem 10. The six schemes correspond to the six ways in which the q-multinomial coefficient $\left[\begin{array}{c}i+j+k \\ i, j, k\end{array}\right]$ can be expanded in terms of

$$
\left[\begin{array}{c}
i+j+k-1 \\
i-1, j, k
\end{array}\right], \quad\left[\begin{array}{c}
i+j+k-1 \\
i, j-1, k
\end{array}\right], \quad\left[\begin{array}{c}
i+j+k-1 \\
i, j, k-1
\end{array}\right] .
$$


In particular, under the standard transformations, the six companions functions $S_{\mu}, \mu=1,2, \cdots, 6$, to Schur's partition function $S(n)$ correspond to these six recurrences for the q-multinomial coefficients.

\section{CONSEQUENCES OF THE BIJECTIONS}

In $\S 4$ we constructed bijections $\mathscr{F}_{\mu, \omega}, 1 \leq \mu, \omega \leq 6$, which converted partitions $\pi_{\mu}$ of Type $\mu$ to partitions $\pi_{\omega}$ of Type $\omega$. That is,

$$
\mathscr{F}_{\mu, \omega}\left(\pi_{\mu}\right)=\pi_{\omega}
$$

and

$$
\mathscr{F}_{\mu, \omega}^{-1}\left(\pi_{\omega}\right)=\mathscr{F}_{\omega, \mu}\left(\pi_{\omega}\right)=\pi_{\mu} .
$$

The following are obvious invariants under the mappings $\mathscr{F}_{\mu, \omega}$. For all $\mu$ and $\omega$ :

$$
\begin{aligned}
\sigma\left(\pi_{\mu}\right) & =\sigma\left(\pi_{\omega}\right), \\
\nu_{a}\left(\pi_{\mu}\right)=\nu_{a}\left(\pi_{\omega}\right), & \nu_{b}\left(\pi_{\mu}\right)=\nu_{b}\left(\pi_{\omega}\right), \quad \nu_{c}\left(\pi_{\mu}\right)=\nu_{c}\left(\pi_{\omega}\right) .
\end{aligned}
$$

In addition there are other invariants depending on the values $\mu$ and $\omega$.

For instance, under the map $\mathscr{F}_{1,2}$ discussed in $\S 3$, the positions occupied by the $a$-parts do not change. Also the weights of the $a$-parts remains invariant under $\mathscr{F}_{1,2}$. In view of this, it is convenient to introduce the function $F_{\mu}\left(x_{m}^{(\mu)} ; a, b, c\right)$, which is the generating function for all Type $\mu$ partitions whose parts are $\preceq x_{m}^{(\mu)}$. (Recall that $x_{m}^{(\mu)}$ denotes the symbol occupying the position $m$ in Scheme $\mu$.) That is,

$$
F_{\mu}\left(x_{m}^{(\mu)}\right)=F_{\mu}\left(x_{m}^{(\mu)} ; a, b, c\right)=\sum_{\substack{\pi_{\mu} \text { of Type } \mu \\ \lambda\left(\pi_{\mu}\right) \preceq x_{m}^{(\mu)}}} a^{\nu_{a}\left(\pi_{\mu}\right)} b^{\nu_{b}\left(\pi_{\mu}\right)} c^{\nu_{c}\left(\pi_{\mu}\right)} q^{\sigma\left(\pi_{\mu}\right)} .
$$

Therefore the invariance of the positions and weights of the $a$-parts under the map $\mathscr{F}_{1,2}$, yields

$$
F_{1}\left(a_{m} ; a, b, c\right)=F_{2}\left(a_{m} ; a, b, c\right) .
$$

Next, given a Type 1 partition $\pi_{1}$ whose parts are $\preceq a_{m}$, we see that either $\lambda\left(\pi_{1}\right)=a_{m}$ or $\lambda\left(\pi_{1}\right) \preceq c_{m}$. If $\lambda\left(\pi_{1}\right)=a_{m}$, then by deleting this part we get a Type 1 partition with largest part $\preceq a_{m-1}$. Thus from (7.2) we see that

$$
F_{1}\left(a_{m} ; a, b, c\right)=a q^{a_{m}} F_{1}\left(a_{m-1} ; a, b, c\right)+F_{1}\left(c_{m} ; a, b, c\right) .
$$

By means of a similar analysis of Type 2 partitions we have

$$
F_{2}\left(a_{m} ; a, b, c\right)=a q^{a_{m}} F_{2}\left(a_{m-1} ; a, b, c\right)+F_{2}\left(b_{m-1} ; a, b, c\right) .
$$

Now (7.3), (7.4) and (7.5) yield

$$
F_{1}\left(c_{m} ; a, b, c\right)=F_{2}\left(b_{m-1} ; a, b, c\right) \text {. }
$$

There is a nice explanation for the equalities (7.3) and (7.6). The $a_{m}$ occupy position $3 m-2$ is Scheme 1 and Scheme 2. Also, the set of symbols $\preceq a_{m}$ in Scheme 1 equals the set of symbols $\preceq a_{m}$ in Scheme 2. This is why (7.3) holds. Similarly, the $c_{m}$ in Scheme 1 and $b_{m-1}$ in Scheme 2 occupy position $3 m-3$. Also the set of symbols $\preceq c_{m}$ in Scheme 1 equals the set of symbols 
$\preceq b_{m-1}$ in Scheme 2, explaining the validity of (7.6). At this stage it is worth pointing out that

$$
F_{1}\left(b_{m-1} ; a, b, c\right) \neq F_{2}\left(c_{m} ; a, b, c\right),
$$

even though $b_{m-1}$ in Scheme 1 and $c_{m}$ in Scheme 2 occupy position $3 m-1$. This is because the set of symbols $\preceq b_{m-1}$ in Scheme 1 is not equal to the set of symbols $\preceq c_{m}$ in Scheme 2 .

Analogous to (7.3) we have

$$
\begin{aligned}
F_{1}\left(c_{m+1} ; a, b, c\right) & =F_{4}\left(c_{m+1} ; a, b, c\right), \\
F_{2}\left(b_{m} ; a, b, c\right) & =F_{3}\left(b_{m} ; a, b, c\right), \\
F_{5}\left(a_{m} ; a, b, c\right) & =F_{6}\left(a_{m} ; a, b, c\right) .
\end{aligned}
$$

To prove (7.7), observe that the positions occupied by the $c_{m}$ remain invariant under $\mathscr{F}_{1,4}$. Similarly, to get (7.8) note that under the map $\mathscr{F}_{2,3}$, the positions occupied $b_{m}$ do not change. Finally, (7.9) follows from the invariance of positions occupied by $a_{m}$ under $\mathscr{F}_{5,6}$. In addition to these, observe also that the positions occupied by $c_{m+1}, b_{m}$ and $a_{m}$ in (7.7), (7.8) and (7.9) are all equal to $3 \mathrm{~m}$. Upon comparing (7.6), (7.7) and (7.8) we arrive at

$$
F_{1}\left(c_{m+1} ; a, b, c\right)=F_{2}\left(b_{m} ; a, b, c\right)=F_{3}\left(b_{m} ; a, b, c\right)=F_{4}\left(c_{m+1} ; a, b, c\right) \text {. }
$$

Next, observe that under the mapping $\mathscr{F}_{3,6}$ the positions occupied by $c_{m+1}$ remains invariant. Therefore

$$
F_{3}\left(c_{m+1} ; a, b, c\right)=F_{6}\left(c_{m+1} ; a, b, c\right) \text {. }
$$

In the lexicographic ordering of Schemes 3 and 6 , the $c_{m+2}$ occupy position $3 m+1$. The symbols occupying the positions $3 m$ in Schemes 3 and 6 are $b_{m}$ and $a_{m}$ respectively (see (4.3) and (4.6)). Therefore, in analogy with (7.4) and (7.5) we have

$$
F_{3}\left(c_{m+2} ; a, b, c\right)=c q^{m+2} F_{3}\left(b_{m-1} ; a, b, c\right)+F_{3}\left(b_{m} ; a, b, c\right)
$$

and

$$
F_{6}\left(c_{m+2} ; a, b, c\right)=c q^{m+2} F_{6}\left(a_{m-1} ; a, b, c\right)+F_{6}\left(a_{m} ; a, b, c\right) .
$$

Hence from (7.11), (7.12) and (7.13), we see by induction on $m$ that

$$
F_{3}\left(b_{m} ; a, b, c\right)=F_{6}\left(a_{m} ; a, b, c\right) \text {. }
$$

Finally, combining (7.9), (7.10) and (7.14), we obtain

$$
\begin{aligned}
& \quad F_{1}\left(c_{m+1} ; a, b, c\right)=F_{2}\left(b_{m} ; a, b, c\right)=F_{3}\left(b_{m} ; a, b, c\right) \\
& \quad=F_{4}\left(c_{m+1} ; a, b, c\right)=F_{5}\left(a_{m} ; a, b, c\right)=F_{6}\left(a_{m} ; a, b, c\right) .
\end{aligned}
$$

Having established (7.15), we may explain these equalities by observing that the symbols $a_{m}$ in Schemes 5 and $6, b_{m}$ in Schemes 2 and 3 , and $c_{m+1}$ in Schemes 1 and 4 all occupy the same position, namely $3 \mathrm{~m}$. In addition, the set of symbols in all six schemes are the same when truncated at position $3 \mathrm{~m}$. That is,

$$
\begin{aligned}
& \left\{x_{1}^{(\mu)}, x_{2}^{(\mu)}, \cdots, x_{3 m}^{(\mu)}\right\} \\
& \quad=\left\{a_{1}, a_{2}, \cdots, a_{m}, b_{1}, \cdots, b_{m}, c_{2}, \cdots, c_{m+1}\right\}, \\
& \quad \mu=1,2, \cdots, 6 .
\end{aligned}
$$

Thus (7.15) may be recast in the following more elegant form. 
Theorem 11. With $F_{\mu}\left(x_{m}^{(\mu)}\right)$ as in (7.2) we have

$$
F_{1}\left(x_{3 m}^{(1)}\right)=F_{2}\left(x_{3 m}^{(2)}\right)=\cdots=F_{6}\left(x_{3 m}^{(6)}\right) .
$$

It is to be noted that the proof of Theorem 11 and (7.15) given above makes use of the bijections $\mathscr{F}_{\mu, \omega}$ and recurrences like (7.3), and is not via (7.16). It might be worthwhile to give a purely algebraic proof of Theorem 11 based on (7.16).

Under the standard transformations something quite striking happens in (7.16), namely, all the sets are equal to

$$
\{1,2,3, \cdots, 3 m\} \text {. }
$$

That is, the set of symbols $\preceq x_{3 m}^{(\mu)}$ in Scheme $\mu$ is equal to the set of positive integers $\leq 3 m$. Thus, if $K_{\mu}(m ; a, b, c)=K_{\mu}(m)$ represents the $a-b-c-q$ generating function of all partitions of Type $\mu$ with the additional restriction that all parts are $\leq m$, then we have

Theorem 12. $K_{1}(3 m)=K_{2}(3 m)=\cdots=K_{6}(3 m)$.

Theorem 2 is a corollary of Theorem 12 by setting $a=b=c=1$ and comparing the coefficients of $q^{n}$. In addition, Theorem 8 is a consequence of Theorem 12 by letting $n \rightarrow \infty$. Thus Theorems 11 and 12 yield substantial generalizations and refinements of Theorems $\mathrm{A}$ and $\mathrm{S}$.

\section{CONCLUDING REMARKS}

The method of weighted words provided the general setting from which Schur's theorem, the companion results, the refinements involving parameters $a, b$ and $c$, and generalizations to an arbitrary modulus $M \geq 3$ all emerged. We have been able to extend the method and apply it to other partition problems as well.

For Schur's theorem we started out with two primary colors $a$ (red) and $b$ (blue), and one secondary color $c=a b$ (purple). In a subsequent paper written jointly with Andrews [2], we view deep theorems of Göllnitz ([13], Sätze 4.8 and 4.10) as emerging out of a situation where we have three primary colors $a, b$ and $c$, and three secondary colors $a b, a c$ and $b c$. Indeed, Schur's theorem corresponds to the special case obtained by setting $c=0$, in which case $c, a c$ and $b c$ drop out and only $a, b$ and $a b$ survive.

Motivated by a study of Lie algebras, Capparelli [12] recently conjectured a partition theorem for which Andrews [9] gave a proof using generating functions. In a joint paper written with Andrews [3], we obtain refinements and generalizations of Capparelli's theorem by the method of weighted words involving free parameters $a, b$ and $c$. The bijections discussed in $\S 3$ can be modified to apply to the Capparelli problem.

\section{ACKNOWLEDGEMENTS}

The first author acknowledges with pleasure the hospitality extended by the mathematics department of Pennsylvania State University and especially by Professor George Andrews during his sabbatical visit in 1992-93 when this work was done. He would like to thank George Andrews for drawing attention to Theorem A and David Bressoud for discussions relating to Scheme 3. Indeed, 
it was the effort to understand the relationship between Theorem $A$ and Theorem $\mathrm{S}$ that led to this paper.

\section{REFERENCES}

1. K. Alladi and B. Gordon, Generalizations of Schur's partition theorem, Manuscripta Math. 79 (1993), 113-126.

2. K. Alladi, G. E. Andrews and B. Gordon, Generalizations and refinements of a partition theorem of Göllnitz, J.Reine Angew. Math. (to appear).

3. $\ldots$, Refinements and generalizations of Capparelli's conjecture on partitions, J. Algebra (to appear).

4. G. E. Andrews, The theory of partitions, Encyclopedia of Math., vol. 2, Addison-Wesley, Reading, MA, 1976.

5. __ On Schur's second partition theorem, Glasgow Math. J. 9 (1967), 127-132.

6. $\ldots$ A new generalization of Schur's second partition theorem, Acta Arith. 4 (1968), 429-434.

7. $\ldots$ A general partition theorem with difference conditions, Amer. J. Math. 191 (1969), 18-24.

8. in Number Theory (A. O. L. Atkin and B. J. Birch, eds.), Academic Press, New York, 1971, pp. 377-387.

9. Schur's theorem, Capparelli's conjecture and q-trinomial coefficients, The Rademacher Legacy to Mathematics (Proc. Rademacher Centenary Conf, 1992), Contemp. Math., Amer. Math. Soc., Providence, RI, 1994, pp. 141-154.

10. D. M. Bressoud, On a partition theorem of Göllnitz, J. Reine Angew. Math. 305 (1979), 215-217.

11. $\_$A combinatorial proof of Schur's 1926 partition theorem, Proc. Amer. Math. Soc. 79 (1980), 338-340.

12. S. Capparelli, On some representations of twisted affine Lie algebras and combinatorial identities, J. Algebra 154 (1993), 335-355.

13. H. Göllnitz, Partitionen mit Differenzenbedingungen, J. Reine Angew. Math. 225 (1967), 154-190.

14. I. J. Schur, Zur additiven Zahlentheorie, Gessammelte Abhandlungen, vol. 2, SpringerVerlag, Berlin, 1973, pp. 43-50.

Department of Mathematics, University of Florida, Gainesville, Florida 32611

E-mail address: alladi@math.ufl.edu

Department of Mathematics, University of California, Los Angeles, California 90024

E-mail address: bg@math.ucla.edu 